\title{
First-Principles Computation of the Vibrational Entropy of Ordered and Disordered $\mathrm{Ni}_{3} \mathrm{Al}$
}

\author{
A. van de Walle and G. Ceder \\ Department of Materials Science and Engineering, Massachusetts Institute of Technology, Cambridge, Massachusetts 02139 \\ U. V. Waghmare \\ Department of Physics, Harvard University, Cambridge, Massachusetts 02138
}

(Received 21 August 1997)

\begin{abstract}
There is increasing evidence that vibrational entropy may significantly contribute to the entropy difference between the ordered and the disordered states of a compound. Through first-principles calculations, we investigate the magnitude of this vibrational entropy difference in $\mathrm{Ni}_{3} \mathrm{Al}$, a compound where this effect is believed to be especially large. We find the vibrational entropy difference to be essentially zero and temperature independent. [S0031-9007(98)06236-X]

PACS numbers: 64.70.Kb, 63.20.-e, 65.50. $+\mathrm{m}$
\end{abstract}

First-principles theory of order-disorder phase equilibria is a well-established field [1,2]. Typically, only configurational entropy is accounted for, and the effect of lattice vibrations on the free energy difference between the ordered and the disordered states is neglected, although no formal justification of this assumption has ever been presented. On the contrary, recent experimental data [3-6] seem to indicate that, in many systems, the vibrational entropy difference between the ordered and the disordered states is comparable to the configurational entropy difference. An especially striking example is the $\mathrm{Ni}_{3} \mathrm{Al}$ compound, for which the vibrational entropy difference was estimated from experimental observations to be $0.2 k_{B}$ [4], whereas the configurational entropy difference is at most $0.57 k_{B}$. If this estimate is correct, it may explain why most first-principles calculations including only configurational entropy consistently overestimate phase transition temperatures. Based on the experimental data of Fultz and co-workers [4], Garbulsky [7] predicted that vibrational effects would shift down the calculated order-disorder transition temperature of $\mathrm{Ni}_{3} \mathrm{Al}$ by $18 \%$ from what it would be with a configurational-only entropy model.

In order to unambiguously confirm that lattice vibrations indeed play an important role, this experimental evidence needs to be backed by a suitable theoretical investigation. Unfortunately, highly accurate first-principles calculations of the vibrational entropy are very computationally intensive tasks. In this context, several investigators turned to simplified models to estimate the vibrational entropy. In some studies $[8,9]$, the complexity of the first-principles approach was tackled by using a simplified model for the vibrational entropy based on the Debye-Gruneisen approximation. All of these results seem to indicate that vibrational effects can be non-negligible.

Calculations of the vibrational entropy difference between disordered and ordered $\mathrm{Ni}_{3} \mathrm{Al}$ (hereafter denoted $\Delta S_{\mathrm{vib}}^{o \rightarrow d}$ ) has so far been performed using only the semiempirical embedded atom method (EAM) [10-12]. Although the specific result seems to depend somewhat on the EAM potential used, all authors found values between $0.1 k_{B}$ and $0.3 k_{B}$, which corresponds to the range of values found experimentally.

Although the value of $\Delta S_{\mathrm{vib}}^{o \rightarrow d}$ calculated through EAM agrees with experimental results, there is no consensus on the origin of this difference. The EAM results indicate that disordering causes a nearly uniform softening of all phonon modes due to an increase in volume. On the other hand, it has been argued from neutron scattering experiments [4] that the vibrational entropy increase is mainly due to a significant decrease of the number of high frequency vibrational modes. Surprisingly, the samples used to measure vibrational entropy differences even exhibited a decrease in lattice constant upon disordering, in contrast to the EAM results.

Some EAM investigations [11] found that a large fraction of $\Delta S_{\mathrm{vib}}^{O \rightarrow d}$ is due to thermal expansion differences between the ordered and the disordered states, while experiments [3,4], as well as some theoretical investigations [12], obtained a large $\Delta S_{\mathrm{vib}}^{o \rightarrow d}$ without considering this effect. Finally, recent EAM calculations [13] (which update some of the results found in [10]) found essentially no contribution from thermal expansion.

The purpose of this paper is to attempt to reconcile some of these conflicting observations and to assess the importance of lattice vibrations in the $\mathrm{Ni}-\mathrm{Al}$ system through a first-principles calculation of the difference in vibrational entropy between the ordered and disordered states for the $\mathrm{Ni}_{3} \mathrm{Al}$ compound. We find that this entropy difference is small $\left[(0.00 \pm 0.05) k_{B}\right]$ and essentially temperature independent $\left[(0 \pm 1) \times 10^{-5} k_{B} \mathrm{~K}^{-1}\right]$.

The absence of thermal expansion effects in our findings corroborates recent EAM calculations [13] and validates the previous investigations which neglected that effect $[3,4,12]$. However, the small magnitude of the vibrational entropy difference we predict is in clear contrast with previous semiempirical calculations and experiments. While this result does not rule out the possibility that lattice vibrations play a significant role in other systems, it does 
point out that vibrational effects in $\mathrm{Ni}_{3} \mathrm{Al}$ may be much smaller than originally claimed.

We will first outline the methodology used in our investigation, leaving the details of the computations for a forthcoming article. We will then provide plausible explanations for the larger value of the vibrational entropy difference obtained in previous experimental and computational studies.

As in other computational investigations [10], we use the quasiharmonic approximation [14]: Lattice vibrations are considered harmonic, but their frequencies are volume dependent. Under these assumptions, the vibrational entropy $S_{\text {vib }}(V)$ can be obtained from the phonon density of states (DOS) $g(V, \nu)$ at volume $V$. The equilibrium volume at temperature $T$ is found by minimizing the free energy $F=E(V)-T S_{\text {vib }}(V)$ with respect to $V$. We thus need to compute $S_{\text {vib }}$ from the phonon DOS at various cell volumes and parametrize $S_{\mathrm{vib}}$ as a function of $V$. At each volume, all internal degrees of freedom as well as the cell shape are relaxed (at constant volume) before computing $S_{\mathrm{vib}}$.

Following the method described in Refs. [15,16], we compute the phonon DOS of a structure by perturbing atoms from their equilibrium position and fitting the spring constants of a Born-von Kármán model to the reaction forces. The Born-von Kármán model can then be used to compute the phonon DOS. The precision of this approach can be controlled by gradually including longerranged spring interactions until the value of vibrational entropy converges (see Table I). In our case, even a first nearest neighbor spring model gives us an accuracy of $0.025 k_{B}$. The use of such a small range of interactions is not unusual: It has been observed [15] that even though a long-range spring model is required to model all the features of the phonon DOS, an integrated quantity such as the vibrational entropy converges much faster with respect to the range of interaction included. It is important to note that once convergence with respect to interaction range is reached, this approach should be just as reliable as the well known linear response method [17-19], since both methods rely on the same assumption of harmonicity. We choose to use a Born-von Kármán model because it typically has lower computational requirements when convergence is reached with a short-range spring model.

Our first-principles computations are performed within the local density approximation (LDA), using the soft pseudopotential approximation and a plane wave basis [20-24]. Spin polarization is neglected in all calculations,

TABLE I. Vibrational Entropy (in $k_{B}$ ) as a function of the number of nearest neighbor (NN) shell included in the Bornvon Kármán Model.

\begin{tabular}{lccc}
\hline \hline Structure & First NN shell & Second NN shell & Third NN shell \\
\hline L1 $_{2}$ & -5.550 & -5.576 & -5.575 \\
SQS-8 & -5.569 & & \\
\hline \hline
\end{tabular}

since the Curie temperature of $\mathrm{Ni}_{3} \mathrm{Al}$ is only $41 \mathrm{~K}$ [25], while the temperature range of interest is above $600 \mathrm{~K}$.

Figure 1 shows how our calculated phonon DOS of the $\mathrm{L1}_{2}$ structure compares with experimental results [4]. Because of the fact that LDA systematically underestimates lattice constants, the calculated frequencies are slightly too high. However, since a similar effect is present for both the ordered and the disordered states, this bias is expected to mostly cancel out. Our model predicts a linear thermal expansion coefficient of $13.2 \times 10^{-6} \mathrm{~K}^{-1}$ for ordered $\mathrm{Ni}_{3} \mathrm{Al}$ in the high-temperature limit. This compares well with experimental measurements [26] which find it to vary from $13.9 \times 10^{-6}$ to $14.9 \times 10^{-6} \mathrm{~K}^{-1}$ between 600 and $900 \mathrm{~K}$.

While the determination of the vibrational entropy of the ordered $\mathrm{L}_{2}$ structure is straightforward, computational resources prohibit the use of large supercells to approximate the random state. The disordered state is thus approximated by a special quasirandom structure (SQS) [27]. SQS structures are the periodic structures that best approximate the disordered state in a unit cell of a given size and have been very successfully used to obtain electronic and thermodynamic properties of disordered materials (see, for example, [28]). We use an 8 atom SQS that possesses the same nearest neighbor pair correlation as the disordered state (see Table II); that is, every $\mathrm{Al}$ atom in the SQS is, on average, surrounded by the same number of $\mathrm{Al}$ atom as in the true disordered state (and similarly for $\mathrm{Ni}$ atoms). The correlations of the SQS, given in Table II, are defined as follows: Spinlike variables are assigned to each site of the lattice $(-1$ for $\mathrm{Ni}$ and +1 for $\mathrm{Al})$. The correlation associated with a given cluster of sites (e.g., a pair of neighboring sites) is then obtained by taking the product of the spins of each site of this cluster and by averaging this quantity over all clusters which are equivalent by the symmetry of the parent lattice.

In order to verify that correlations other than the nearest neighbor pair do not have a significant influence on the vibrational entropy, we compute the vibrational entropy difference between two structures $\left(\mathrm{L1}_{2}\right.$ and $\left.\mathrm{DO}_{22}\right)$

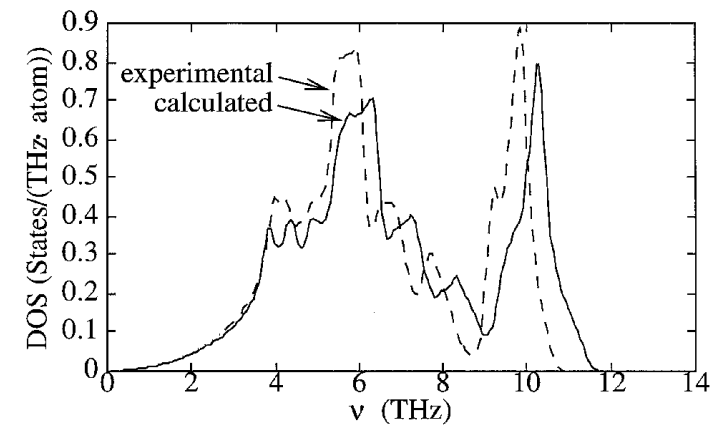

FIG. 1. Calculated and experimental phonon DOS of the $\mathrm{L}_{2}$ structures. The experimental phonon DOS is calculated from the force constant provided in [4], which are themselves fitted from previous phonon dispersion measurement [38]. 
TABLE II. Correlations of the structures used. $p_{n}$ denotes the $n$th nearest neighbor correlation while $t_{l m n}$ denotes a triplet made of overlapping $p_{l}, p_{m}$, and $p_{n}$ pairs.

\begin{tabular}{lcccccc}
\hline \hline Structure & $p_{1}$ & $p_{2}$ & $t_{111}$ & $t_{112}$ & $t_{113}$ & $t_{114}$ \\
\hline $\mathrm{L}_{2}$ & 0 & 1 & $1 / 2$ & $-1 / 2$ & $1 / 2$ & $-1 / 2$ \\
$\mathrm{DO}_{22}$ & 0 & $2 / 3$ & $1 / 2$ & $-1 / 6$ & $1 / 6$ & $1 / 6$ \\
$\mathrm{SQS}-8$ & $1 / 4$ & $1 / 3$ & $-1 / 4$ & 0 & $-1 / 12$ & $-1 / 6$ \\
Random & $1 / 4$ & $1 / 4$ & $-1 / 8$ & $-1 / 8$ & $-1 / 8$ & $-1 / 8$ \\
\hline \hline
\end{tabular}

which share the same nearest neighbor correlation but differ by their other correlations. Our first-principles calculations show this difference to be at most $0.04 k_{B}$ for temperatures between 600 and $2000 \mathrm{~K}$. The difference in the correlations between the SQS and the disordered state are at least a factor of 2 smaller than the difference in correlation between the $\mathrm{L}_{2}$ and $\mathrm{DO}_{22}$ structures (see Table II). We can thus reasonably expect that a difference of $0.04 k_{B}$ between the $\mathrm{L}_{2}$ and $\mathrm{DO}_{22}$ structures translates into a correspondingly smaller entropy difference between the SQS structure and the disordered state, that is, an error of at most $0.02 k_{B}$.

The main result of these calculations is that we find almost no difference in vibrational entropy between the ordered and the disordered states $\left[(0.00 \pm 0.05) k_{B}\right]$. The error bracket is found by adding the uncertainties due to using only nearest neighbor springs for the SQS $\left(0.025 k_{B}\right)$ and due to using a SQS having only the correct nearest neighbor pair correlations $\left(0.02 k_{B}\right)$. We do find a large temperature dependence of $S_{\mathrm{vib}}$ for both the ordered and the disordered states (about $2.4 \times 10^{-4} k_{B} \mathrm{~K}^{-1}$ ). However, since the difference in temperature dependence between the two states is very small $(0 \pm 1 \times$ $\left.10^{-5} k_{B} \mathrm{~K}^{-1}\right), \Delta S_{\mathrm{vib}}^{o \rightarrow d}$ remains small at all temperatures.

How can we explain the apparent discrepancy between our findings $\left[(0.00 \pm 0.05) k_{B}\right]$ and results from inelastic neutron scattering [4] (from $0.1 k_{B}$ to $0.3 k_{B}$ )? Although this range of experimental results is often averaged to give $0.2 k_{B}$, the true result is probably closer to $0.1 k_{B}$. The higher bound of $0.3 k_{B}$ was obtained using the virtual crystal approximation to analyze the neutron scattering data of the disordered state, while the lower bound of $0.1 k_{B}$ was obtained when the DOS of the disordered state was assumed to resemble that of the ordered state. Recent EAM calculations [10,11], as well as our own results from the SQS calculations (see Fig. 2), clearly show the latter hypothesis to be the correct one: The DOS of the disordered state is a broadened version of the DOS of the ordered state. The experimental result is therefore likely to be close to the lower bound of $0.1 k_{B}$. The remaining discrepancy between our calculation and experiment can well be accounted for by other sources of entropy in the experiment.

As $\mathrm{Ni}_{3} \mathrm{Al}$ is ordered up to the melting point, disordered $\mathrm{Ni}_{3} \mathrm{Al}$ needs to be produced by vapor deposition [29] or ball milling [30]. Both of these methods produce a

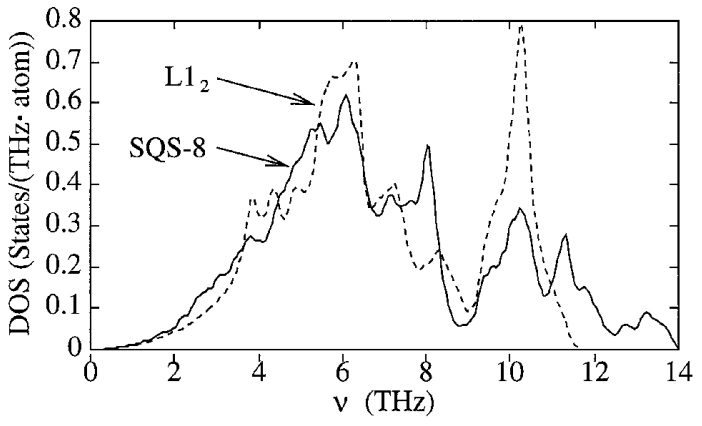

FIG. 2. Calculated phonon DOS of the $\mathrm{L}_{2}$ and SQS-8 structures.

material with a very small grain size (respectively, $4 \mathrm{~nm}$ [29] and $7 \mathrm{~nm}$ [31]). When the samples are annealed to reach the ordered state, the grain size inevitably increases. It follows that the ordered and disordered samples differ not only by their state of order, but also by their grain size. Grain size can have a significant effect on the thermodynamics of nanocrystalline materials. The latter typically possess higher heat capacities than their coarsegrained counterparts due to the large fraction of atoms lying in the grain boundary regions which are typically softer [31-33]. For example, it has been observed that the vibrational entropy difference between disordered $\mathrm{Ni}_{3} \mathrm{Fe}$ in its nanocrystalline (9 $\mathrm{nm}$ average grain size) and coarse-grained form is about $0.18 k_{B}$ [31], which is of the same order of magnitude as $\Delta S_{\mathrm{vib}}^{o \rightarrow d}$ in $\mathrm{Ni}_{3} \mathrm{Al}$.

This small grain size effect is visible in the vibrational DOS obtained through neutron scattering. It is responsible for the enhancement of the density of low frequency phonon modes [4]. We obtain an estimate of this effect by integrating the experimentally measured $\ln (\nu)\left[g^{o}(\nu)-g^{d}(\nu)\right]$ over the low frequency part of the DOS. To yield a meaningful value, this integral has to be taken over a range of frequencies which encloses the same number of modes for both the ordered and the disordered states. From the data of Fultz et al. (Ref. [4], Fig. 4), we obtain a low frequency contribution of about $0.05 k_{B}$ by integrating from 0 to $22 \mathrm{meV}$. Frequencies above $22 \mathrm{meV}$ provide the remaining $0.05 k_{B}$ attributable to the order-disorder transition. With this new interpretation, the neutron scattering results now lie between the EAM and the $a b$ initio predictions.

In calorimetric measurements [3], grain size effects are expected to be even larger, as the grain size was only $4 \mathrm{~nm}$ (about 20 atomic layers), compared to $7 \mathrm{~nm}$ in neutron scattering experiments. This partly explains the larger value of the vibrational entropy difference found with calorimetric measurements.

The effect of small grain size can also explain the disagreement between the calculated and experimental lattice parameters. All calculations indicate that the disordered state has a larger volume than the ordered state $(1 \%-2 \%$ larger with EAM, $0.5 \%$ larger with $a b$ initio). On the 
contrary, some experiments [4] (but not all, see [34]) find that the ordered state has the largest lattice constant. The difference arises from the fact that the lattice constant of nanocrystalline materials can often differ by $\pm 0.3 \%$ from their bulk counterparts [35-37]. This effect can dominate over the volume expansion of the bulk material only if the latter is not too large. In this sense, the small volume expansion upon disordering we obtain is more consistent with experimental observations than the EAM results. This small volume change upon disordering is in part responsible for our small value of $\Delta S_{\mathrm{vib}}^{o \rightarrow d}$.

In conclusion, we find a remarkably small value of $\Delta S_{\mathrm{vib}}^{o \rightarrow d}$, in contrast to interpretations from previous experiments and several semiempirical calculations. However, in light of our results, the upper and the lower bounds on $\Delta S_{\mathrm{vib}}^{o \rightarrow d}$ obtained from neutron scattering data should be reinterpreted. The upper bound, based on the virtual crystal approximation, is unlikely to be appropriate, while the lower bound is probably much closer to the actual $\Delta S_{\mathrm{vib}}^{o \rightarrow d}$. After small grain size effects are taken into account, the resulting $\Delta S_{\mathrm{vib}}^{o \rightarrow d}$ (about $0.05 k_{B}$ ) lies between the EAM predictions $\left(0.1 k_{B}\right.$ to $\left.0.3 k_{B}\right)$ and the $a b$ initio predictions $\left[(0.00 \pm 0.05) k_{B}\right]$. The difference between our $a b$ initio calculations and the EAM results originates from the fact that we find a smaller volume expansion upon disordering $(0.5 \%)$. Finally, we find no significant effect of thermal expansion on $\Delta S_{\mathrm{vib}}^{o \rightarrow d}$ in $\mathrm{Ni}_{3} \mathrm{Al}$.

Understanding the magnitude of the vibrational entropy variations between different states of order remains a central problem in first-principles alloy theory. This variation had been thought to be especially large in $\mathrm{Ni}_{3} \mathrm{Al}$, a perception with which our results disagree. It remains of interest to evaluate the magnitude of this effect in other systems.

This work was sponsored in part by the National Science Foundation under Contract No. DMR9501856 and by the Petroleum Research Fund, Grant No. ACSPRF29133-AC5. Axel van de Walle acknowledges support from a "1967" scholarship from the Natural Sciences and Engineering Research Council of Canada. The authors thank G. Garbulsky for providing computer programs which made this work possible.

[1] F. Ducastelle, Order and Phase Stability in Alloys (Elsevier Science, New York, 1991).

[2] D. de Fontaine, Solid State Phys. 47, 33 (1994).

[3] L. Anthony, J. K. Okamoto, and B. Fultz, Phys. Rev. Lett. 70, 1128 (1993).

[4] B. Fultz et al., Phys. Rev. B 52, 3315 (1995).

[5] L. Anthony, L. J. Nagel, J. K. Okamoto, and B. Fultz, Phys. Rev. Lett. 73, 3034 (1994).
[6] L. J. Nagel, L. Anthony, and B. Fultz, Philos. Mag. Lett. 72, 421 (1995).

[7] G. D. Garbulsky and G. Ceder, Phys. Rev. B 53, 8993 (1996).

[8] J. M. Sanchez, J.P. Stark, and V.L. Moruzzi, Phys. Rev. B 44, 5411 (1991).

[9] M. Asta, R. McCormack, and D. de Fontaine, Phys. Rev. B 48, 748 (1993).

[10] J. D. Althoff et al., Phys. Rev. B 56, R5705 (1997).

[11] R. Ravelo et al., Phys. Rev. B 57, 862 (1998).

[12] G. J. Ackland, in Alloy Modelling and Design, edited by G. Stocks and P. Turchi (The Minerals, Metals and Materials Society, Pittsburgh, PA, 1994), pp. 149-153.

[13] D. Morgan (private communication).

[14] A. A. Maradudin, E. W. Montroll, and G. H. Weiss, Theory of Lattice Dynamics in the Harmonic Approximation, Second Edition (Academic Press, New York, 1971).

[15] G.D. Garbulsky, Ph.D. thesis, Massachusetts Institute of Technology, 1996.

[16] S. Wei and M. Y. Chou, Phys. Rev. Lett. 69, 2799 (1992).

[17] X. Gonze, D. C. Allan, and M. P. Teter, Phys. Rev. Lett. 68, 3603 (1992).

[18] S. de Gironcoli, Phys. Rev. B 51, 6773 (1995).

[19] U. V. Waghmare, Ph.D. thesis, Yale University, 1996.

[20] G. Kresse and J. Furthmüller, Phys. Rev. B 54, 11169 (1996).

[21] G. Kresse and J. Furthmüller, Comput. Mater. Sci. 6, 15 (1996).

[22] D. Vanderbilt, Phys. Rev. B 41, 7892 (1990).

[23] J.C. Phillips and L. Kleinman, Phys. Rev. 116, 287 (1959).

[24] M. C. Payne et al., Rev. Mod. Phys. 64, 1045 (1992).

[25] J. Xu, B. I. Min, A. J. Freeman, and T. Oguchi, Phys. Rev. B 41, 5010 (1990).

[26] P. U. T. P. R. Center, in Thermal Expansion: Metallic Elements and Alloys, Thermophysical Properties of Matter, TPRC Data Series Vol. 12, edited by Y.S. Touloukian et al. (IFI/Plenum, New York, 1970-1979).

[27] A. Zunger, S.-H. Wei, L. Ferreira, and J. E. Bernard, Phys. Rev. Lett. 65, 353 (1990).

[28] K. C. Hass, L. C. Davis, and A. Zunger, Phys. Rev. B 42, 3757 (1990).

[29] S. R. Harris, D. H. Pearson, C. M. Garland, and B. Fultz, J. Mater. Res. 6, 2019 (1991).

[30] J. S. C. Jang and C. C. Koch, J. Mater. Res. 5, 498 (1990).

[31] H. N. Frase, L. J. Nagel, J. L. Robertson, and B. Fultz, Philos. Mag. B 75, 335 (1997).

[32] J. Trampenau, K. Bauszus, W. Petry, and U. Herr, Nanostruct. Mater. 6, 551 (1995).

[33] B. Fultz et al., J. Appl. Phys. 79, 8319 (1996).

[34] F. Cardelli et al., J. Mater. Res. 8, 2504 (1993).

[35] I. V. Alexandrov and R.Z. Valiev, Philos. Mag. B 73, 861 (1996).

[36] V. I. Zubov, Nanostruct. Mater. 3, 189 (1993).

[37] K. Lu, H. Y. Zhang, Y. Zhong, and H. J. Fecht, J. Mater. Res. 12, 923 (1997).

[38] C. Stassis, F. X. Kayser, C.-K. Loong, and D. Arch, Phys. Rev. B 24, 3048 (1981). 